Supporting Information for:

\title{
An All-in-One Aptasensor Integrating Enzyme Powered Three-Dimensional DNA Machine for the Antibiotic Detection
}

Tai Ye, Zhiwei Zhang, Min Yuan, Hui Cao, Fengqin Yin, Xiuxiu Wu and Fei Xu*

School of Medical Instrument and Food Engineering, University of Shanghai for Science and Technology, Shanghai 200093, China.

E-mail address: xufei8135@126.com 


\section{Supplementary Experimental Section}

Chemicals and Materials: All oligonucleotides used in this study were synthesized and HPLC purified by Sangon Biotech Co., Ltd. (Shanghai, China). Sequences of the synthesized oligonucleotides are list in Supporting Information Table S1. Gold chloride $\left(\mathrm{HAuCl}_{4} 3 \mathrm{H}_{2} \mathrm{O}\right)$, Sodium citrate $\left(\mathrm{Na}_{3} \mathrm{C}_{6} \mathrm{H}_{5} \mathrm{O}_{7} \cdot 2 \mathrm{H}_{2} \mathrm{O}\right)$, Tween 20, dithiothreitol (DTT), tris(2-carboxyethyl) phosphine hydrochloride $(\mathrm{TCEP} \cdot \mathrm{HCl})$, Kanamycin sulfate were purchased from Sigma-Aldrich (St. Louis, MO). Tetracycline, Chloramphenicol, Ampicillin, Oxytetracycline, streptomycin and Amikacin were brought from Macklin Inc. (Shanghai, China). Nicking endonuclease (Nt.AlwI) and CutSmart buffer (NEB buffer) were ordered from New England Biolabs, Inc. (Beverly, MA, USA). All chemical reagents were of analytical grade and used without further purification. The milk sample were acquired from local supermarket in Shanghai, China. All solutions were prepared with ultra-pure water $(18.25 \mathrm{M} \Omega \cdot \mathrm{cm})$ from a Millipore system.

Apparatus: The transmission electron microscopy (TEM) images were obtained by a JEM-2100 transmission electron microscope (JEOL, Japan). Zeta potential and dynamic light scattering (DLS) were recorded on the Malvern Zetasizer Nano ZS90 (Malvern, USA). The fluorescence spectra and the real-time fluorescence dates were measured on RF-6000 spectrometer with $10 \mathrm{~nm}$ band-pass spectrometer slits (Shimadzu, Japan). The UV-vis absorption spectra were recorded on TU-1901 spectrometer (Persee, Beijing, China). 
Table S1. Sequence information for oligonucleotides used in this work

\begin{tabular}{cl}
\hline Name & Sequence (5'-3') \\
\hline F-probe & HS-TTTTTTTTTTGGATCATAT*AGTAGT-FAM (*cleavage site) \\
Kana-Aptamer & TGGGGGTTGAGGCTAAGCCGA \\
Walker ( 20nt $)$ & HS-T-20-TCGGCTTAGCTACTATATGATCCGAACCCCCA \\
Walker ( 40nt $)$ & HS-T-40-TCGGCTTAGCTACTATATGATCCGAACCCCCA \\
Walker ( 60nt $)$ & HS-T-60-TCGGCTTAGCTACTATATGATCCGAACCCCCA \\
Walker ( 70nt $)$ & HS-T-70-TCGGCTTAGCTACTATATGATCCGAACCCCCA \\
\hline
\end{tabular}



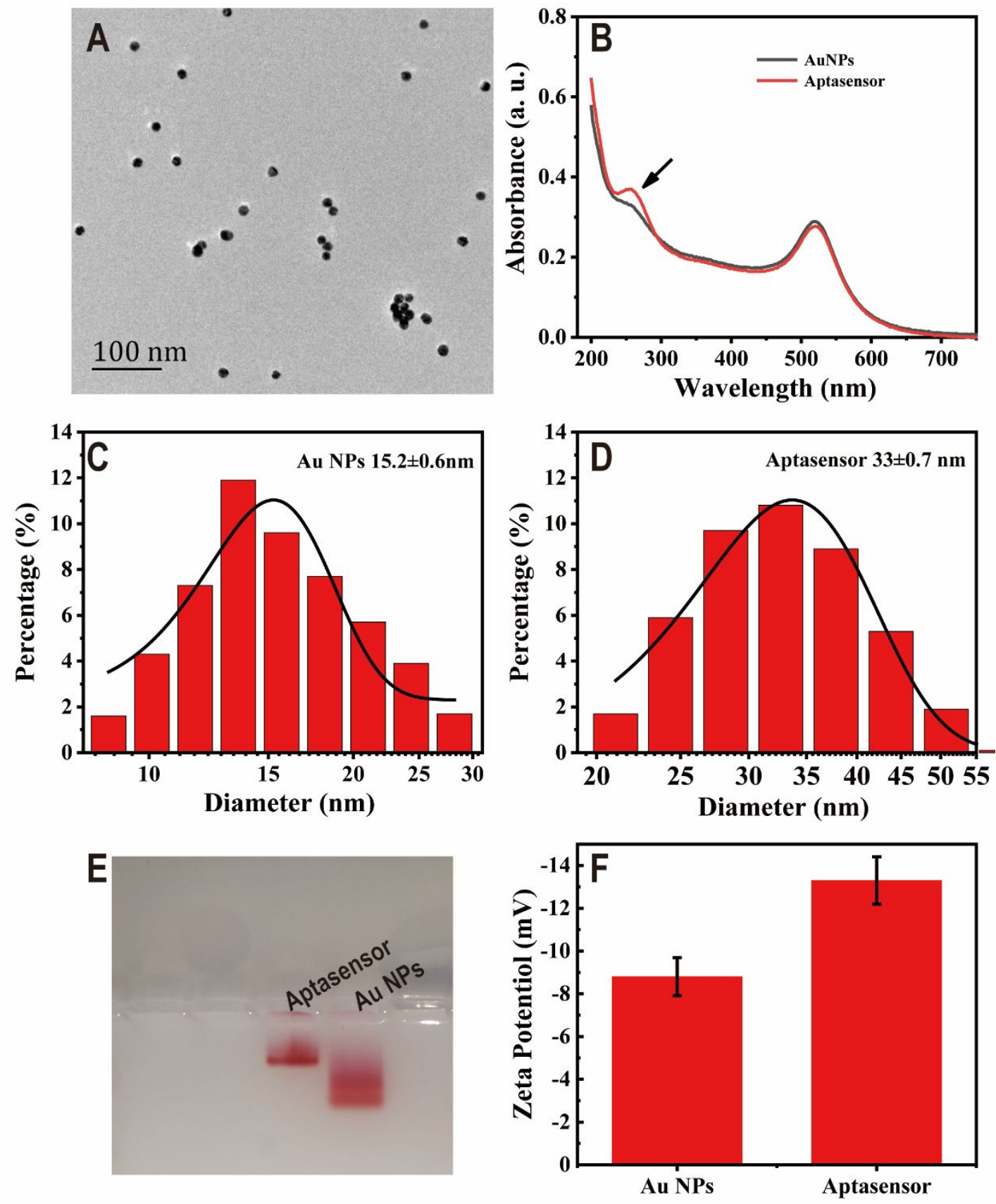

Fig. S1. Characterization of the bare Au NPs and the Aptasensor. TEM images of (A) bare Au NPs.

(B) UV-vis absorption spectra of bare AuNPs (black) and aptasensor (red). Dynamic light scattering (DLS) measurements of (C) bare AuNPs and (D) aptasensor. (E) Agarose gel electrophoresis (F) Zeta-potential of bare AuNPs and aptasensor. 

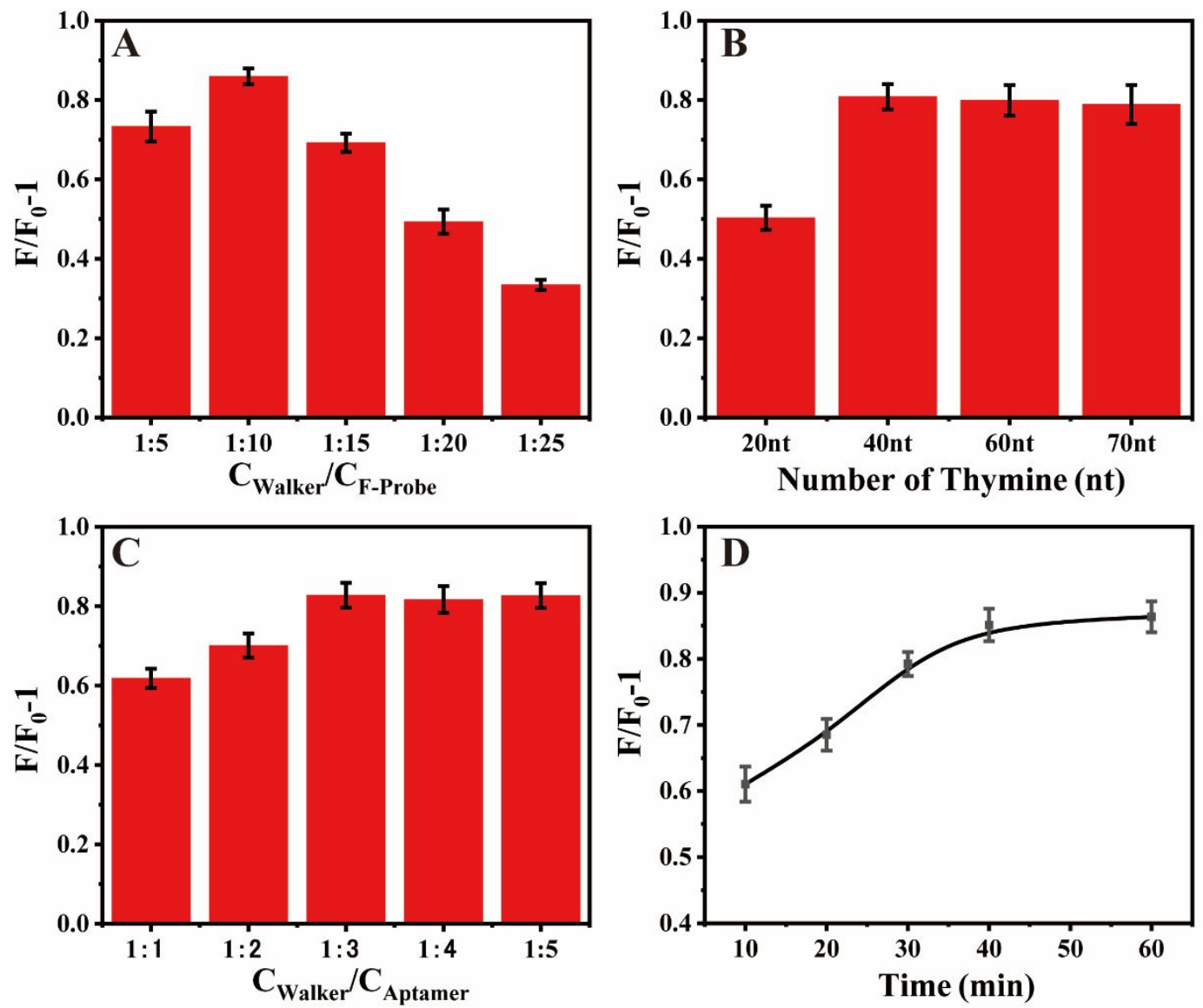

Fig. S2. The influence of the ratio of walker and F-probe (A), the number of thymine (B), the ratio of walker and aptamer (C), and the reaction time (D) on the all-in-one aptasensor. The concentration of kanamycin and Nt.AIwI were $0.5 \mathrm{nM}$ and $10 \mathrm{U}$, respectively. 